\title{
Two components for one resistivity in $\mathrm{LaVO}_{3} / \mathrm{SrTiO}_{3}$ heterostructure.
}

\author{
H. Rotella, ${ }^{1,2}$ O. Copie, ${ }^{1,3}$ A. Pautrat,, , $*$ P. Boullay, ${ }^{1}$ A. David, ${ }^{1}$ \\ D. Pelloquin, ${ }^{1}$ C. Labbé, ${ }^{4}$ C. Frilay, ${ }^{4}$ and W. Prellier ${ }^{1}$ \\ ${ }^{1}$ Laboratoire CRISMAT, CNRS UMR 6508, ENSICAEN et Université de Caen, \\ 6 Bd Maréchal Juin, 14050 Caen Cedex 4, France. \\ ${ }^{2}$ NUSNNI-NanoCore, National University of Singapore, Singapore 117411. \\ ${ }^{3}$ CEA, DSM/IRAMIS/SPEC, F-91191 Gif-sur-Yvette Cedex, France. \\ ${ }^{4}$ Laboratoire CIMAP, CNRS UMR 6252, CEA, ENSICAEN et Université de Caen, \\ 6 Bd Maréchal Juin, 14050 Caen Cedex 4, France.
}

(Dated: September 19, 2018)

\begin{abstract}
A series of $100 \mathrm{~nm} \mathrm{LaVO}_{3}$ thin films have been synthesized on (001)-oriented $\mathrm{SrTiO}_{3}$ substrates using the pulsed laser deposition technique, and the effects of growth temperature are analyzed. Transport properties reveal a large electronic mobility and a non-linear Hall effect at low temperature. In addition, a cross-over from a semiconducting state at high-temperature to a metallic state at low-temperature is observed, with a clear enhancement of the metallic character as the growth temperature increases. Optical absorption measurements combined with the two-bands analysis of the Hall effect show that the metallicity is induced by the diffusion of oxygen vacancies in the $\mathrm{SrTiO}_{3}$ substrate. These results allow to understand that the film/substrate heterostructure behaves as an original semiconducting-metallic parallel resistor, and electronic transport properties are consistently explained.

PACS numbers: $81.15 . \mathrm{Fg}, 68.55 . \mathrm{A}-$, 61.05.cp
\end{abstract}

\section{A. Introduction.}

The discovery of unexpected conducting or superconducting behaviors when stacking two band insulators like $\mathrm{LaAlO}_{3}$ (LAO) and $\mathrm{SrTiO}_{3}(\mathrm{STO})$ has motivated intense activities to understand these phenomena ${ }^{1-3}$. The origin of the highly mobile charge carriers appears the central point of the debate. Interpretations are based on an electronic reconstruction at the interface due to a polar discontinuity between the two insulators ${ }^{2}-5$, on cation intermixing or on oxygen vacancies ${ }^{6}-\frac{8}{-}$. It has also been shown that $2 \mathrm{D}$ electronic conduction can be observed for peculiar controlled conditions. In particular, the oxygen pressure used during the film growth and the annealing conditions are critical parameters. They allow to tune the amount of oxygen vacancies and the dimensionality of transport properties 9,10 . Using a Mott insulator having a strong coulomb interaction instead of a band insulator should allow to take advantage of the rich properties of electronic correlated systems ${ }^{11}$, and other systems such as $\mathrm{LaTiO}_{3} / \mathrm{SrTiO}_{3}$ (LTO/STO) have been investigated 12,13 . Similarly to LAO/STO, LTO/STO displays a conducting behavior which was explained by an electronic reconstruction, and superconductivity emerges at low temperature 13 . $\mathrm{LaVO}_{3} / \mathrm{SrTiO}_{3}(\mathrm{LVO} / \mathrm{STO})$ is another interesting oxide heterostructure, LVO being a Mott insulator that exhibits an antiferromagnetic spin order ${ }^{14}$. Besides the existence of a $2 \mathrm{D}$ electron gaz proposed at the $\mathrm{LVO} / \mathrm{STO}$ interface ${ }^{15}$, new phenomena could arise from the combination of confined carriers at the interface and strong correlations in LVO. Thin films of LVO/STO were synthesized using the pulsed laser deposition (PLD) technique by Hotta et al. $\stackrel{16}{ }$. Importantly, a pure LVO phase can be formed only under low par- tial pressure of oxygen (typ. $<10^{-5}$ Torr), and a postannealing under oxygen should be avoided since the oxidized form of $\mathrm{LaVO}_{4}$ is quickly stabilized. Therefore, the problematic of oxygen vacancies is specially relevant for LVO/STO. Regarding the physical properties, this heterostructure presents a conducting behavior and a low temperature non linear Hall effect, both explained by the intrinsic mechanism of electronic reconstruction at the interface 15 . The primary role of interface effects was also recently proposed to explain the LVO/STO metallic properties $\frac{17}{}$, and the presence of oxygen vacancies was not considered as a single STO substrate placed in the deposition chamber was not conducting after the ablation process. However, this experiment is not sufficient to rule out the formation of oxygen vacancies in a heterostructure. For example in LAO/STO, the presence of oxygen vacancies in STO is strongly enhanced by the presence of a LAO epilayer due to the difference of activation energy for oxygen diffusion between the two materials 7 . The process of doping is then mainly driven by oxygen transfer between an oxygen deficient growing film and its substrate 18 . Similar argument could a priori be applied to the $\mathrm{LVO} / \mathrm{STO}$ case $\stackrel{19}{ }$. Recently, the LVO/STO heterostructure was shown to be a potential efficient solar cell by Assmann et $a^{20}$. In this approach, the presence of an effective electric field due to a polar discontinuity at the interface between the two material is an essential ingredient. It is then important to have a better understanding of the conducting mechanisms in this system.

Here, we address how the growth conditions affect the transport properties in a series of LVO/STO films. Since the pressure can not be significantly changed to stabilize $\mathrm{V}^{3+}$ in $\mathrm{LVO}$, the growth temperature is the most accurate parameter to be considered. We have synthe- 
sized a series of high quality LVO thin films deposited on (001)-oriented STO substrates at different growth temperatures, and investigated both the structural and magnetotransport properties. We show that the transport properties are dominated by a semiconducting-metallic competition which is sensitive to the growth temperature. This is explained by considering the contribution of the STO substrate which is doped by thermally activated oxygen vacancies. This appears to be a generic process in STO based heterostructure.

\section{B. Experimental.}

Epitaxial $\mathrm{LaVO}_{3}$ (LVO) thin films were prepared by the pulsed laser deposition technique on (001)-oriented $\mathrm{SrTiO}_{3}$ (STO) substrates (cubic with $a=3.905 \AA$ ). We have used as-received substrates: No specific treatment has been performed to select the $\mathrm{SrO}$ or $\mathrm{TiO}_{2}$ substrate termination. Note that as-received substrates are $\mathrm{TiO}_{2}$ terminated $^{22,23}$. A KrF laser $(\lambda=248 \mathrm{~nm})$ with a repetition rate of $2 \mathrm{~Hz}$ was focused onto a $\mathrm{LaVO}_{4}$ polycrystalline target at a fluence of $\simeq 2 \mathrm{~J} / \mathrm{cm}^{2}$. The substrate was at a temperature ranging from $600-750^{\circ} \mathrm{C}$ under a dynamic vacuum around $10^{-5} \mathrm{mbar}$ (base pressure). The target-substrate distance was fixed at $8.5 \mathrm{~cm}$. The number of pulses was adjusted to obtain the desired thickness of $100 \mathrm{~nm}^{21}$. The X-ray diffraction measurements were performed with a $\theta / 2 \theta$ diffractometer Seifert XRD 3000P $\left(\lambda_{C u}=1.5406 \AA\right)$ for room temperature measurements and with a $\theta / 2 \theta$ X'pert Pro MPD PANalytical $\left(\lambda_{C o}=1.789 \AA\right)$ for measurements at different temperatures. LVO is orthorhombic (Pnma (\#62)) in its bulk form at room temperature and it undergoes a structural transition below $140 \mathrm{~K}$ into a monoclinic $\left(P 2_{1} / c(\# 11)\right)$ symmetry $\stackrel{24}{\underline{2}}$. Previously, we have shown that similar LVO thin film grown at $700^{\circ} \mathrm{C}$ presents a distorted orthorhombic structure due to the compressive stress induced by STO, with original $\mathrm{VO}_{6}$ rotations ${ }^{21}$. Transport and galvanomagnetic properties were measured using a Quantum Design PPMS. Electrical contacts were realized using $\mathrm{Al} / \mathrm{Au}$ wire-bonding. Sheet resistivity is defined as $\mathrm{R}_{s}=\rho / \mathrm{t}, \rho$ being the bulk resistivity and $t$ the thickness. The absorbance spectra were obtained using a Perkin Elmer spectrophotometer Lambda 1050.

\section{Results and discussion.}

Figure 1a depicts the $\theta$-2 $\theta$ X-ray diffracted pattern of the LVO films grown at various temperatures. The thickness was first defined by the number of laser pulses and was checked to be $t=100 \mathrm{~nm}$ for all the series by fitting the diffracted patterns using the DIFFaX software ${ }^{25}$. The sharpness of the peaks and the presence of Laue fringes confirm the high structural quality and a low interface roughness in agreement with previous reports 26,27 . As the growth temperature decreases, the peak associated to LVO shifts towards smaller $2 \theta$ angle, indicating an increase of the out-of-plane parameter (Fig.1b). From reciprocal space mapping, we know that our LVO films are coherently in plane- strained all over the thickness 21 , and the expanded c-axis corresponds to an increase of the volume. Such expansion of the c-axis parameter can be induced by oxygen vacancies during the film deposition as seen previously in copper oxides films $\stackrel{28}{ }$, STO/STO homoepitaxial films ${ }^{29}$ and recently observed in $\mathrm{PrVO}_{3}$ films grown on $\mathrm{STO}^{30}$.

The sheet resistance $\mathrm{R}_{s}$ of the films was measured as a function of the temperature $T$ (See Fig.2). LVO is a localized system with semiconducting conductivity 31 even in the presence of some cationic and/or oxygen vacancies 32,33 while pure stoichiometric STO is insulating. However, a clear metallic behavior with $(\mathrm{dR} / \mathrm{dT})>0$ is clearly observed up to a temperature $T^{*}$. For $T>T^{*}$, $\mathrm{dR} / \mathrm{dT}<0$ indicates a semiconducting-like behavior. $\mathrm{T}^{*}$ varies from samples to samples with different temperature growth. The room temperature value of the sheet resistance $\mathrm{R}_{s}$ varies from $\mathrm{R}_{s} \sim 2.71$ to $28.5 k \Omega / \square$ with a minimum $\sim 100 \Omega / \square$ at low temperature for the most conducting sample. These values are in good agreement with those reported in other heterostructures (LTO/STO $\stackrel{13}{ }$, LAO/STO ${ }^{3}$ ), where low dimensional conduction and superconductivity have been reported. They are also close to values measured in $\mathrm{LVO} / \mathrm{STO}^{15,17}$. An upturn of the resistance at low temperatures was noted by Hotta et al. and explained by an incipient localization mechanism 15 . Here, this effect is not seen in our measurements. This is possibly due to a smaller amount of defects (such as grain boundaries) in agreement with the lowest residual resistivity $(\sim 100 \Omega / \square$ for the most conducting film).

To understand the origin of the change in resistivity at $\mathrm{T}^{*}$, we focus on the film grown at $700^{\circ} \mathrm{C}$ (Fig.3) with $\mathrm{T}^{*} \sim 225 K$. Since the film has a distorted orthorhombic structure with significant tilts of $\mathrm{VO}_{6}$ octahedra at room temperature $\stackrel{21}{\underline{2}}$ it is worth asking wether the thermal variation of transport properties correlates with changes in lattice parameters and/or structural distortion ${ }^{34}$. In particular, it is not known if the orthorhombic to monoclinic structural phase transition observed at $140 \mathrm{~K}$ in bulk samples ${ }^{24}$ is still observed in the LVO thin film. Note, however that such transition favors orbital ordering, and a more localized state at low temperature, $\stackrel{35}{=}$ which is hardly compatible with the metallicity observed here. The evolution of a-axis and c-axis lattice parameters of the film were recorded as function of temperature under cooling conditions (Fig. 3b and 3c). While a small curvature is observed over a broad temperature range for the c-axis parameter, there is no discontinuous changes that could indicate a transition, and the temperature of c-axis minimum is shifted by $60 \mathrm{~K}$ lower than $\mathrm{T}^{*}(\mathrm{Fig}$. $3 \mathrm{~b})$. We conclude that the apparent semiconductingmetal transition is unrelated to the structural evolution of the LVO film. To go further, we have investigated in details the galvanomagnetic properties of the same sam- 
ple. The magnetoresistance (MR) and Hall effect are measured at different fixed temperatures. As shown in Fig. 4, a positive MR is observed at low temperature which reaches $\operatorname{MR}(14 \mathrm{~T})=\frac{R(14 T)-R(0 T)}{R(0 T)} \sim 0.01$ at $10 \mathrm{~K}$. MR increases continuously when decreasing the temperature, as expected for a metallic MR driven by mean free path effects. This increase is more pronounced for $\mathrm{T} \lesssim$ $100 \mathrm{~K}$, an effect that will be discussed hereafter. Note that a drastic change of MR can be expected at a metal-toinsulator transition due to Fermi surface reconstruction. Here, we observe that the sign of MR changes at high temperature, but only continuously, and at a temperature larger than $T^{*}$ (see inset of Fig. 4). This confirms that $T^{*}$ corresponds to a smooth cross-over between two conducting regimes rather than to a genuine transition.

We have measured the Hall resistance $\mathrm{R}_{x y}$ at various temperatures. Its sign is typical of n-type carriers. A preliminary analysis was made by fitting the data in the low field regime (i.e. where $\mathrm{R}_{x y}$ is a linear function of the applied field) using a single-band model. The mobility $\mu$ and the sheet carriers density $n_{s}$ have been calculated this way as a function of the temperature and the results are reported in Fig. 5. We found $\mu \approx 1.310^{3} \mathrm{~cm}^{2} / \mathrm{V}$.s and $n_{s} \approx 2.10^{13} / \mathrm{cm}^{2}$ at low temperature. The electronic mobility is consistent with the high values reported in other STO based heterostructures, including LVO/STO deposited under low pressure, but are also observed in single phase STO doped by oxygen vacancies, as already discussed for the LAO/STO case $\mathrm{6}^{6.8}$.

When looking closely at the $\mathrm{R}_{x y}(\mathrm{~B})$ curves, a nonlinear field variation of the Hall resistance is evidenced. The non-linearity is more important for $\mathrm{T} \lesssim 100 \mathrm{~K}$. A non-linear Hall resistance was observed by Hotta et al.

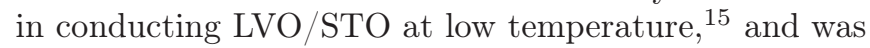
proposed to arise from spin effects such as antiferromagnetic fluctuations at the interface $\frac{15}{\underline{1}}$ or strong spin-orbit coupling 36 . In other heterostructures, a similar nonlinear effect was analyzed with a conventional galvanomagnetic coupling, using a two bands rather than a single band model (in LTO/ST 37,38 , in $\mathrm{LAO} / \mathrm{STO}^{39}$ ).

In a two-bands model, two groups of charge carriers are considered with mobilities $\mu_{1}$ and $\mu_{2}$ and densities $n_{1}$ and $n_{2}$, respectively. In this case, the Hall coefficient $R_{H}=\frac{R_{x y}}{B}$ can be written as $\underline{40}$ :

$$
R_{H}=1 / e \frac{n_{1} \mu_{1}^{2}+n_{2} \mu_{2}^{2}+\left(n_{1}+n_{2}\right) \mu_{1}^{2} \mu_{2}^{2} B^{2}}{\left(n_{1} \mu_{1}+n_{2} \mu_{2}\right)^{2}+\left(n_{1}+n_{2}\right)^{2} \mu_{1}^{2} \mu_{2}^{2} B^{2}}
$$

Due to the four unknown parameters, it is necessary to constraint the fit. We rewrite equation (1) using the approach used in $\underline{41,42}$ :

$$
R_{H}=\frac{R_{0}+R_{\infty} \mu_{*}^{2} B^{2}}{1+\mu_{*}^{2} B^{2}}
$$

which has two fitting parameters $\mu^{*}$ and $R_{\infty}, R_{0}$ being the zero field limit of $R_{H}$ which is graphically determined. With the value of the zero field resistivity
$R_{x x}=\left(e \cdot n_{1} \cdot m u_{1}+e \cdot n_{2} \cdot \mu_{2}\right)^{-1}$, the following equations give four independent parameters $\mu_{1}, \mu_{2}, n_{1}$ and $n_{2}$

$$
\begin{array}{r}
A=\left(R_{0} / R_{x x}+\mu_{*}\right) / 2 \\
\mu_{1}=A+\left(A^{2}-\mu_{*} R_{\infty} / R_{x x}\right)^{1 / 2} \\
\mu_{2}=A-\left(A^{2}-\mu_{*} R_{\infty} / R_{x x}\right)^{1 / 2} \\
C=\left(\mu_{1} / \mu_{2}\right)\left(\mu_{*}-\mu_{2}\right) /\left(\mu_{1}-\mu_{*}\right) \\
n_{1}=\frac{1}{e R_{\infty}(1+C)} \\
n_{2}=\frac{C}{e R_{\infty}(1+C)}
\end{array}
$$

Analysis of the data using this two-bands model is very satisfactory, as demonstrated by the agreement between the experimental data and the modeled curve reported in the Fig.6. The extracted parameters are shown in the Fig.7 for all temperatures. At the lowest temperature, we find carriers densities ratio $n_{1} / n_{2} \approx 0.14$ and mobilities ratio $\mu_{1} / \mu_{2} \approx 3.2$. One open question is whether the two-bands scenario arises from spatially separated carriers with one of the carriers family located at the interface of the heterostructure $\stackrel{38}{ }$. Another possibility is that these different carriers are intrinsic to STO due to its complex electronic band structure with at least the presence of one heavy and one light electron band $\underline{43}-\underline{45}$. The pertinence of a multiband scenario intrinsic to STO was recently confirmed by Laukhin et al based on measurements and analysis of the pressure dependence of electronic mobilities and carrier density $\stackrel{46}{ }$, and by Lin et al $\underline{45}$. From literature, the ratio of effective mass $m_{1} / m_{2}$ is expected $\sim$ 10-20 at low temperature $\underline{47}$. The mobilities are related to the effective masses by $\left(\mu_{2} / \mu_{1}\right) \sim\left(m_{1} / m_{2}\right)^{q}$ with q a constant dependent on the scattering mechanism ${ }^{40}$. Thus, for a dominant charged impurity scattering at low temperature where $\mathrm{q}=1 / 2$, one finds $\left(\mu_{2} / \mu_{1}\right) \sim 3.1-4.4$ in very good agreement with our result. From magnetoresistance and Hall measurements, a ratio $\mu_{1} / \mu_{2} \approx 2.7$ was reported in the low temperature state of n-type $\mathrm{STO}^{48}$. This value is also close to our result. We finally note that both the MR and the non linearity of Hall effect are reinforced for $\mathrm{T}<100 \mathrm{~K}$. This temperature is close to the temperature of the cubic to tetragonal transition of STO, where some changes in its galvanomagnetic properties can be expected $\underline{48}$. To conclude this analysis, the contribution of STO itself is sufficient to explain the nonlinear Hall effect in LVO/STO, and we propose that this result can be expanded to the case of other STO-based heterostructures.

Since the measure of optical properties can reveal the presence of oxygen vacancies in $\mathrm{STO}^{49}$, we have measured the optical absorption of the sample grown at $700^{\circ} \mathrm{C}$, and of an as-received STO substrate for a reference. As shown in Fig. 8, the absorption spectra of the (film+substrate) structure shows a broad peak centered around $2.4 \mathrm{eV}$, and a smaller one around $1.7 \mathrm{eV}$, whereas both peaks are absent in the virgin substrate. These peaks lying below 
the $3.2 \mathrm{eV}$ band-gap are characteristic of reduced STO bulk crystals, and are systematically attributed to the presence of $\mathrm{F}$ centers caused by oxygen vacancies $\underline{49}$.

Based on the experimental data that evidence the presence of oxygen vacancies in STO, we propose a simple model to quantify this effect and to explain the macroscopic transport properties including the apparent metalto-semiconducting transition. Indeed, the low pressure ( 10 ${ }^{-5}$ mbar) used during the LVO growth induces oxygen vacancies in the deposited layers. These vacancies prefer to migrate inside the STO due to its low activation energy for vacancies diffusion ${ }^{7,19}$. The kinetics of this process is thermally activated, and the relatively high growth temperature $\left(\mathrm{T}_{\text {Growth }}>600^{\circ} \mathrm{C}\right)$ allows for their effective migration during the ablation process. Also, the c-axis parameter is known to expand with the oxygen vacancies (c-axis expansion effect) $\underline{28,30}$. If a hightemperature growth favors oxygen vacancies in the substrate, the deposited LVO film is correlatively more close to its stoichiometry in oxygen. Consistently, we observe a decrease of the c-axis LVO value when the growth temperature increases. Small deviation from perfect La/V ratio can be favored by oxygen deficiency in $\mathrm{LVO}^{31,50}$. However, such non-stoichiometry has only a small effect on the transport properties of LVO which is a robust semiconductor with only small band gap changes 33 . On the contrary, it is well known that STO becomes conducting even with a tiny doping by oxygen vacancies ${ }^{8.10}$. We have then to deal with two resistors in parallel: the LVO film expected to be semiconducting-like in one side, and the STO substrate being partially conducting on the other side. One assumption is that the conducting thickness of the STO is fixed by the diffusion length of oxygen vacancies at the macroscopic scale, and could be strongly dependent on the growth temperature. Note this parallel resistor with opposite temperature variation mimics an equivalent resistor with non-monotonic temperature variation which presents a resistance maximum at some intermediate temperature ${ }^{51}$, in qualitative agreement with our measurements (Fig.2). To be more quantitative, $G_{i}$, $\rho_{i}$ and $t_{i}$ will stand for conductance, resistivity and thickness, respectively, while $\mathrm{i}=1,2$ are the LVO and STO. For a parallel resistor, the equivalent conductance $\mathrm{G}$ is:

$$
G=\frac{1}{R}=G_{1}+G_{2}=\frac{1}{R_{1}}+\frac{1}{R_{2}}=\frac{t_{1} W}{d \rho_{1}}+\frac{t_{2} W}{d \rho_{2}}
$$

While $t_{1}=100 \mathrm{~nm}$ is the physical thickness of LVO, $t_{2}$ is an effective thickness corresponding to the conducting part of STO which is related to the oxygen vacancies diffusion length $\ell, \mathrm{d}$ and $\mathrm{W}$ are respectively the distances between the voltage pads and the film width. For all the samples, the low temperature resistance $R(T)$ can be fitted by $R_{\text {res }}+A . T^{2}$ over a large temperature range $\left(R_{\text {res }}\right.$ is the residual resistance). This is typical of a doped-STO contribution (Fig. 9). This indicates that the conducting paths are short-circuited by the substrate at low temperature $\left(G_{1} \ll G_{2}\right)$ and $R \sim R_{2}$ for $\mathrm{T} \lesssim 100 \mathrm{~K}$. Assuming that $R_{2}(T)=R_{0}+A . T^{2}$ is fulfilled up to $300 \mathrm{~K}$ as in bulk STO, $R_{1}(T)$ and $\rho_{1}(T)$ are directly deduced using equation (4).

The bulk LVO conduction is semiconducting-like with a resistivity described by a thermally activated law: $\rho(T) \propto \exp \left(E_{a} / K T\right)$ (where $E_{a}$ is the activation energy, $K$ the Boltzmann constant). As shown in Fig.10, $R_{1}(T)$ presents such an activated behavior for all the samples. The extracted energies $E_{a}$ are close to $0.2 \mathrm{eV}$, a very reasonable value for conducting processes in rare earth vanadates such as $\mathrm{LVO}^{31}$. The $\rho_{1}$ value taken at room temperature is also close the resistivity of bulk LVO, i.e. in a range 0.1-0.3 $\Omega . \mathrm{cm}, \frac{31}{=}$ and increases when $\mathrm{T}_{\text {growth }}$ decreases (Fig.10). Since the highest growth temperature favors the diffusion of oxygen vacancies in STO, their amount in LVO is expected to be larger at the lowest temperature. The presence of oxygen vacancies or charge defects often reduce the resistance by doping and/or by creating mixed valencies in oxides. In LVO, we observe an increase of resistance when we expect the largest oxygen non-stoichiometry. There is however no contradiction with the literature since the introduction of mixed valency in LVO by non stoichiometry seems to favor the carriers localization with the creation of defect states 33 . Finally, we conclude that $\mathrm{R}_{1}$ has to be associated with the LVO film.

Let us now return to the behavior of STO. The oxygen vacancies diffusion mechanism is a thermally activated process, with a diffusion constant given by an Arrhenius dependence $D(T)=D_{0} \exp (-U / K T)$ where $D_{0}$ is the diffusion constant, $U$ the activation energy. Thus, during a growth time $\tau_{d}$ and at a certain $T_{\text {growth }}$ temperature, the diffusion length in a $1 \mathrm{D}$ diffusion is given by $\ell\left(T_{\text {growth }}\right)=2 \sqrt{\times D\left(T_{\text {growth }}\right) \times \tau_{d}}$. Dealing with the room temperature values of conductivity, we neglect here the possible spreading of carriers due to the unusual large dielectric constant of STO at low temperature ${ }^{8,10}$, and we approximate the effective thickness of conducting STO by $t_{2} \sim \ell$. At room temperature and below, we assume that the thermal energy (typ. $0.026 \mathrm{eV}$ at $300 \mathrm{~K}$ ) is low enough to neglect any supplementary vacancies diffusion after the growth process. Finally, the STO conductance $G_{2}$ can be written as:

$$
G_{2}=1 / R_{2} \sim 2 \sqrt{\times D_{0} \exp \left(U / K T_{\text {growth }}\right) \tau_{d}} / \rho_{2}
$$

Here, the important parameter is $\mathrm{U}$, the activation energy for the diffusion of oxygen vacancies in STO, which is experimentally given by the slope of $\ln \left(G_{2}\right)$ as function of $1 / T_{\text {growth }}$ (Fig. 10). The fit leads to $U \sim 0.9 \mathrm{eV}$, in good agreement with literature $(U \sim 0.7-1.4 \mathrm{eV})^{52,53}$.

In conclusion, we have studied the electronic conduction properties of a series of $\mathrm{LaVO}_{3}$ (LVO) thin films grown at various temperature on $\mathrm{SrTiO}_{3}$ (STO) substrates. A pronounced metallic character is observed as in other heterostructures based on STO. The better metallicity is observed for the films synthetised at the highest growth temperature. We show, thanks to a de- 
tailed analysis of galvanomagnetic and optical properties in one film, that the metallic component arises from the $\mathrm{SrTiO}_{3}$ substrate which was populated by oxygen vacancies during the film growth. As a consequence, the heterostructure can be thought as a parallel resistor with a semiconducting LVO component and a metallic STO component, and the equivalent resistance mimics an apparent metal to semiconducting-like transition. Even though electronic reconstruction effects might be present in the system, we conclude that the macroscopic electronic transport properties are mainly driven by the competition between these two bulk components. At low temperature, STO dominates the galvanomagnetic prop- erties, including the non-linear Hall effect.

We thank Fabien Veillon (CRISMAT) and Jerome Lecourt (CRISMAT) for the precious help in respectively the physical properties measurements, and the ceramic synthesis for thin film growth. Discussions with and encouragement of the emeritus Prof B. Mercey (CRISMAT) were highly appreciated. We thank also Jean Louis Doualan (CIMAP) for technical help for the optical measurements. Partial support of the French Agence Nationale de la Recherche (ANR), through the program Investissements d'Avenir (ANR-10-LABEX-09-01), LabEx EMC3, the C'Nano program and the Interreg IVA MEET project are also acknowledged.
* Electronic address: alain.pautrat@ensicaen.fr Corresponding author

1 A. Ohtomo and H. Y. Hwang, Nature 427, 423 (2004).

2 N. Reyren, S. Thiel, A.D. Caviglia, L.F. Kourkoutis, G. Hammerl, C. Richter, C.W. Schneider, T. Kopp, A.S. Retschi, D. Jaccard, M. Gabay, D.A. Muller, J.M. Triscone, and J. Mannhart, Science 317, 1196 (2007).

3 A.D. Caviglia, S. Gariglio, N. Reyren, D. Jaccard, T. Schneider, M. Gabay, S. Thiel, G. Hammerl, J. Mannhart, and J.-M. Triscone, Nature (London) 456, 624 (2008).

4 N. Nakagawa, H.Y. Hwang, and D.A. Muller, Nat. Mater. 5, 204 (2006).

5 S. Thiel, G. Hammerl, A. Schmehl, C.W. Schneider, and J. Mannhart, Science 313, 1942 (2006).

${ }^{6}$ G. Herranz, M. Basletic, M. Bibes, C. Carretero, E. Tafra, E. Jacquet, K. Bouzehouane, C. Deranlot, A. Hamzic, J.M. Broto, A. Barthelemy, and A. Fert, Phys. Rev. Lett. 98, 216803 (2007).

7 A. Kalabukhov, R. Gunnarsson, J. Borjesson, E. Olsson, T. Claeson, and D. Winkler, Phys. Rev. B 75, 121404(R) (2007)

8 W. Siemons, G. Koster, H. Yamamoto, W. A. Harrison, G. Lucovsky, Th.H. Geballe, D.H.A. Blank, and M.R. Beasley, Phys. Rev. Lett. 98, 196802 (2007).

9 M. Basletic, J.-L. Maurice, C. Carretero, G. Herranz, O. Copie, M. Bibes, E. Jacquet, K. Bouzehouane, S. Fusil and A. Barthelemy, Nature Mater. 7, 621 (2008).

10 O. Copie, V. Garcia, C. Bodefeld, C. Carretero, M. Bibes, G. Herranz, E. Jacquet, J.-L. Maurice, B. Vinter, S. Fusil, K. Bouzehouane, H. Jaffres, and A. Barthelemy, Phys. Rev. Lett. 102, 216804 (2009).

11 S. Okamoto and A.J. Millis, Nature 428, 630 (2004).

12 A. Ohtomo, D.A. Muller, J. L. Grazul, and H. Y. Hwang, Nature 419, 378 (2002).

13 J. Biscaras, N. Bergeal, A. Kushwaha, T. Wolf, A. Rastogi, R.C. Budhani, J. Lesueur, Nature Communications 1, 1 (2010).

14 A.V. Mahajan, D.C. Johnston, D.R. Torgeson, and F. Borsa, Phys. Rev. B 46, 10966 (1992).

15 Y. Hotta, T. Susaki, and H.Y. Hwang, Phys. Rev. Lett. 99, 236805 (2007)

16 Y. Hotta, Y. Mukunoki, T. Susaki, H.Y. Hwang, L. Fitting and D. A. Muller, Appl. Phys. Lett. 89, 031918 (2006).

17 C. He, T. D. Sanders, M.T. Gray, F. J. Wong, V. V. Mehta, and Y. Suzuki, Physical Review B 86, 081401 (2012).
18 W. Schneider, M. Esposito, I. Marozau, K. Conder, M. Doebeli, Yi Hu, M. Mallepell, A. Wokaun and T. Lippert, Appl. Phys. Lett. 97, 192107 (2010).

19 M. Cherry, M.S. Islam and C.R.A. Catlow, J. Solid State Chem. 188, 125 (1995).

20 E. Assmann, P. Blaha, R. Laskowski, K. Held, S. Okamoto, and G. Sangiovanni, Phys. Rev. Lett. 110, 078701 (2013).

${ }^{21}$ H. Rotella, U. Luders, P.-E. Janolin, V. H. Dao, D. Chateigner, R. Feyerherm, E. Dudzik, and W. Prellier, Phys. Rev. B 85, 184101 (2012).

22 G. Koster, B. L. Kropman, G. J. H. M. Rijnders, D. H. A. Blank, and H. Rogalla, Appl. Phys. Lett. 73, 2920 (1998).

23 A. Biswas, P. B. Rossen, C. H. Yang, W. Siemons, M. H. Jung, I. K. Yang, R. Ramesh, and Y. H. Jeong, Appl. Phys. Lett. 98, 051904 (2011).

24 P. Bordet, C. Chaillout, M. Marezio, Q. Huang, A. Santoro, S-W. Cheong, H. Takagi, C.S. Oglesby, B. Batlogg, J. Sol. State Chem. 106, 253 (1993).

25 M. M. J. Treacy, M. W. Deen, DIFFaX. http://www.public.asu.edu/mtreacy/DIFFaX.html

26 P. Boullay, A. David, W. C. Sheets, U. Luders, W. Prellier, H. Tan, J. Verbeeck, G. Van Tendeloo, C. Gatel, G. Vincze, and Z. Radi, Phys. Rev. B 83, 125403 (2011).

27 Haiyan Tan, Ricardo Egoavil, Armand Beche, Gerardo T. Martinez, Sandra Van Aert, Jo Verbeeck, Gustaaf Van Tendeloo, Helene Rotella, Philippe Boullay, Alain Pautrat, and Wilfrid Prellier, Phys. Rev. B 88, 155123 (2013).

28 J.C. Cheang Wong, C. Ortega, J. Siejka, I. Trimaille, A. Sacuto, L.M. Mercandalli, F. Mayca, J. Alloys \& Comp. 195, 675 (1993).

29 A. Ohtomo and H. Y. Hwang, J. Appl. Phys. 102, 083704 (2007)).

30 O. Copie, H. Rotella, P. Boullay, M. Morales, A. Pautrat, P-E Janolin, I. C. Infante, D. Pravathana, U. Luders and W. Prellier, J. Phys.: Condens. Matter 25, 492201 (2013).

31 D. B. Rogers, A. Ferretti, D. H. Ridgley, R. J. Arnott, and J.B. Goodenough, J. Appl. Phys. 37, 1431 (1966).

${ }^{32}$ H. Hur, S. H. Kim, K. S. Yu, Y. K. Park, J. C. Park, and S. J. Kim, Solid State Commun. 92, 541 (1994).

33 S. Jamali Gharetape, M. P. Singh, F. S. Razavi, D. A. Crandles, L. Y. Zhao and K. T. Leung, Appl. Phys. Lett. 98, 052509 (2011).

34 A. David, R. Fresard, Ph. Boullay, W. Prellier, U. Luders and P.-E. Janolin, Appl. Phys. Lett. 98, 212106 (2011).

35 J.S. Zhou, Y. Ren, J.Q. Yan, J.F. Mitchell, and J.B. Good- 
enough, Phys. Rev. Lett. 100, 046401 (2008).

${ }^{36}$ H. Weng and K. Terakura, Phys. Rev. B. 82, 115105 (2010).

37 J. S. Kim, S. S. A. Seo, M. F. Chisholm, R. K. Kremer, H.-U. Habermeier, B. Keimer, and H. N. Lee, Phys. Rev. B 82, 201407 (2010).

38 J. Biscaras, N. Bergeal, S. Hurand, C. Grossetete, A. Rastogi, R.C. Budhani, D. LeBoeuf, C. Proust, and J. Lesueur, Phys. Rev. Lett.108 , 247004 (2012).

39 C. Bell, S. Harashima, Y. Kozuka, M. Kim, B. G. Kim, Y. Hikita, and H. Y. Hwang, Phys. Rev. Lett. 103, 226802 (2009).

40 J. M. Ziman, Principles of the Theory of Solids, Cambridge University Press, (1964).

41 E.K. Arushanov and G.P. Chuiko, Phys. Status Solidi (a) 17, K135 (1973).

42 R. Laiho, A.V. Lashkul, K.G. Lisunov, E. Lahderanta, M.O. Safonchik and M.A. Shakhov, Semicond. Sci. Technol. 19, 602 (2004).

43 L.F. Mattheiss, Phys. Rev. B 6, 4718 (1972).

44 M. Ahrens, R. Merkle, B. Rahmati and J. Maier, Physica B 393, 239 (2007).

45 X. Lin, Z. Zhu, B. Fauque, and K. Behnia, Phys. Rev. X 3, 021002 (2013).

${ }^{46}$ V. Laukhin, O. Copie, M. J. Rozenberg, R. Weht, K. Bouzehouane, N. Reyren, E. Jacquet, M. Bibes, A. Barthelemy, and G. Herranz, Phys. Rev. Lett. 109, 226601 (2012).

47 A.F. Santander-Syro, O. Copie, T. Kondo, F. Fortuna, S. Pailhes, R. Weht, X.G. Qiu, F. Bertran, A. Nicolaou, A. Taleb-Ibrahimi, P. Le Fevre, G. Herranz, M. Bibes, Y. Apertet, P. Lecoeur, M. J. Rozenberg, A. Barthelemy, Nature 469, 189 (2011).

48 F.Kuchar and P. Frankus, Phys. Rev. B 16, 874 (1977).

49 W. S. Baer, Phys. Rev. 144, 734 (1966); C. Lee, J. Destry, and J.L. Brebner, Phys. Rev. B 11, 2299 (1975).

${ }^{50}$ H. Seim and H. Fjellvag, Acta Chem. Scand.52, 1096 (1998).

51 C. Grygiel, A. Pautrat, W. Prellier, B. Mercey, W. C. Sheets, and L. Mechin, J. Phys.: Condens. Matter 20, 472205 (2008).

${ }^{52}$ P. Pasierb, S. Komornicki, and M. Rekas, J. Phys. Chem. Sol. 60, 1835 (1999).

53 R. De Souza, V. Metlenko, D. Park, and T. Weirich, Phys. Rev. B 85, 174109 (2012). 

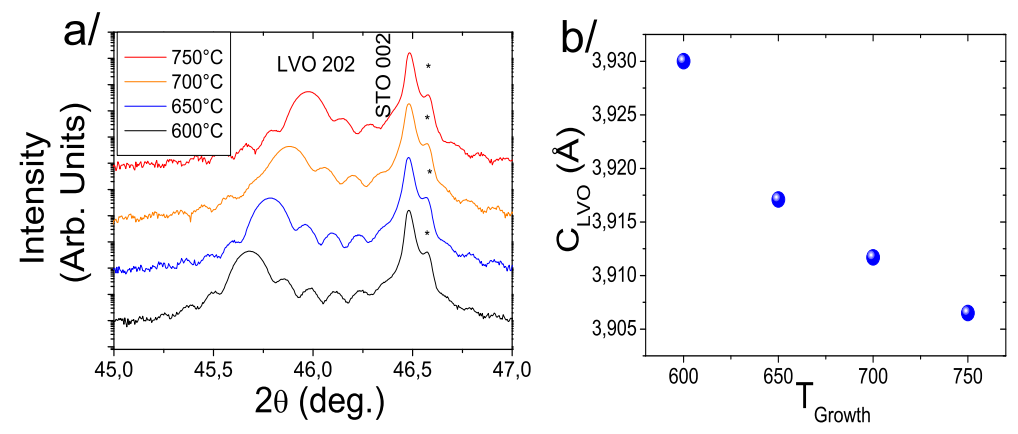

FIG. 1: a/ $\theta-2 \theta$ X-ray diffracted patterns of $100 \mathrm{~nm}$ thick films grown at $600,650,700$ and $750^{\circ} \mathrm{C}$. The star indicates the $\mathrm{K}_{\alpha 2}$ contribution of the (002) $\mathrm{SrTiO}_{3}$ reflection.B/ c-axis pseudo cubic parameter of LVO as function of the growth temperature.

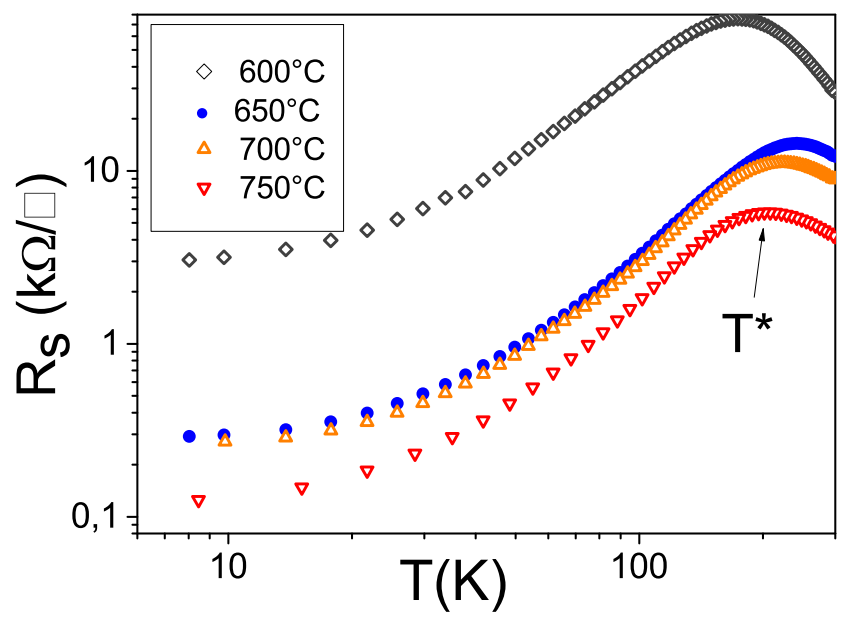

FIG. 2: Sheet resistance $\mathrm{R}_{s}$ of the samples grown at different temperatures in a log-log scale. Note the maximum of resistance at $\mathrm{T}^{*}$. 


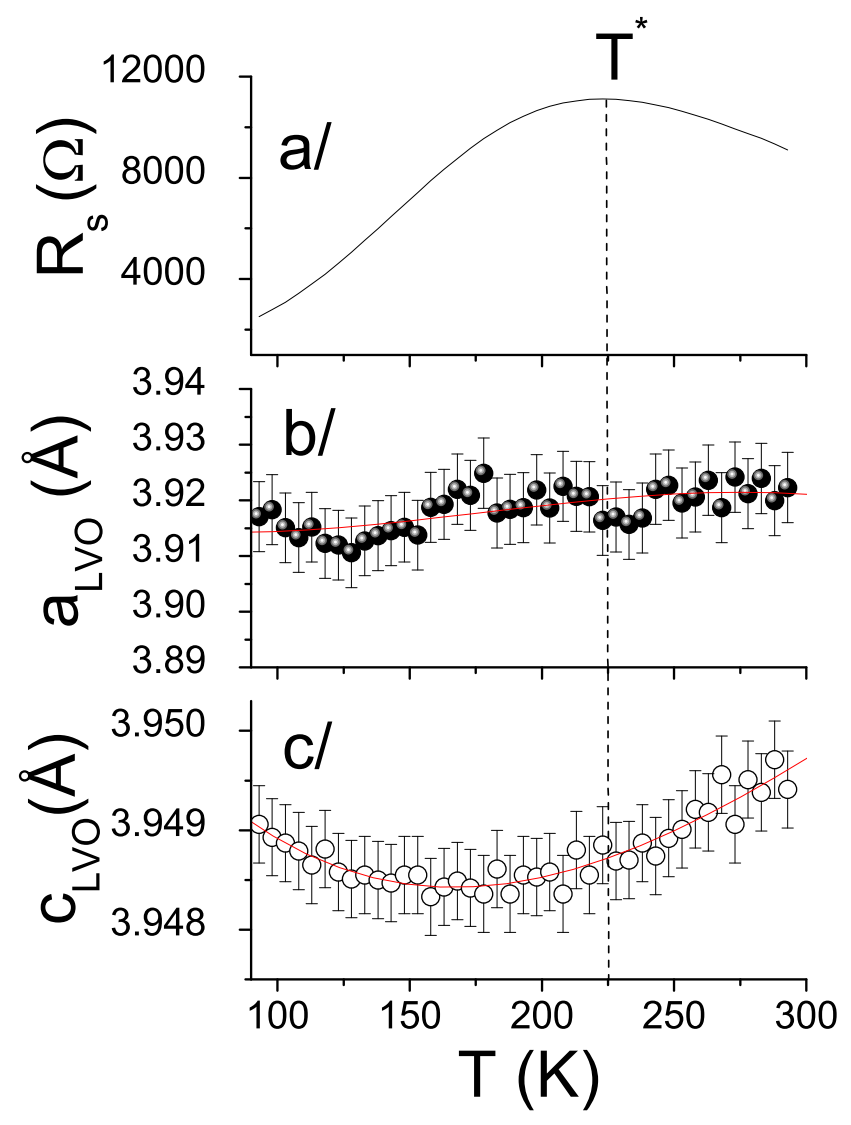

FIG. 3: a/ Variation of the sheet resistance of LVO/STO grown at $700^{\circ}$ as a function of temperature. Note the maximum at a temperature $\mathrm{T}^{*}$ signing a semiconducting-like to metallic change of conductivity. b/ a-axis parameter of the LVO film as a function of the temperature. $\mathrm{c} / \mathrm{c}$-axis parameter of the LVO film as function of temperature. Solid lines are guide for the eyes (polynomial fits). Note the large difference between $\mathrm{T}^{*}$ and the temperature where the c-axis parameter is minimum. 


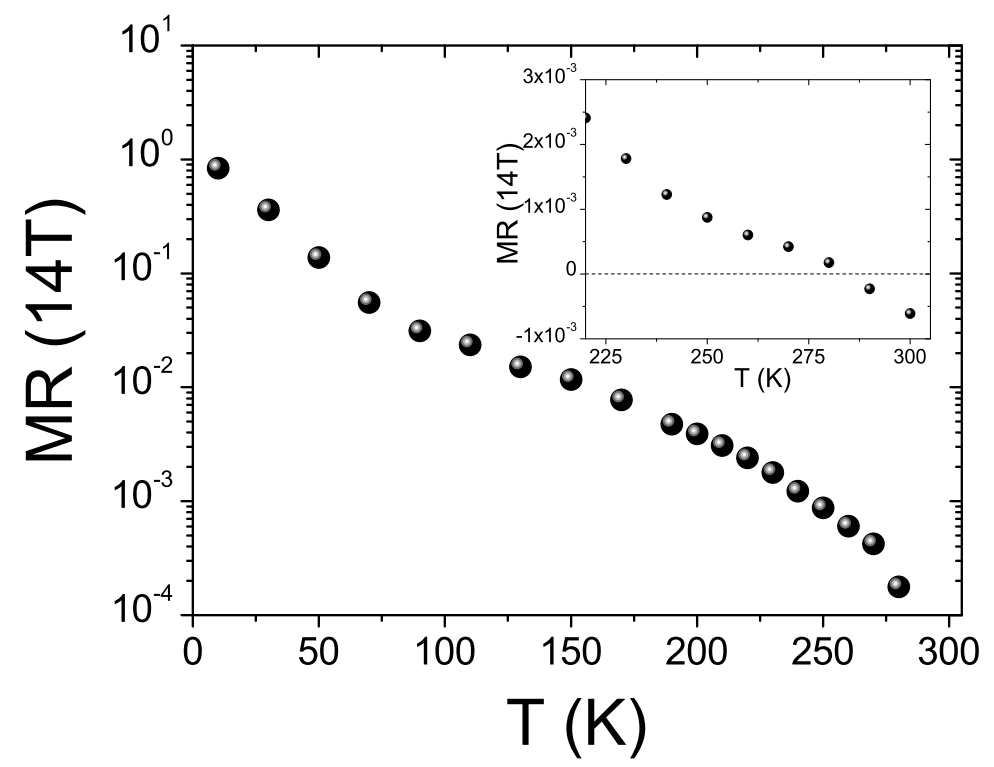

FIG. 4: Magnetoresistance at $14 \mathrm{~T}$ vs. temperature in a loglinear scale for the sample grown at $700^{\circ}$. Note the increase in magnitude for $\mathrm{T} \lesssim 100 \mathrm{~K}$. Insert displays the cross-over between positive and negative $\mathrm{MR}$ at $\mathrm{T}>\mathrm{T}^{*}$ (linear-linear scale).

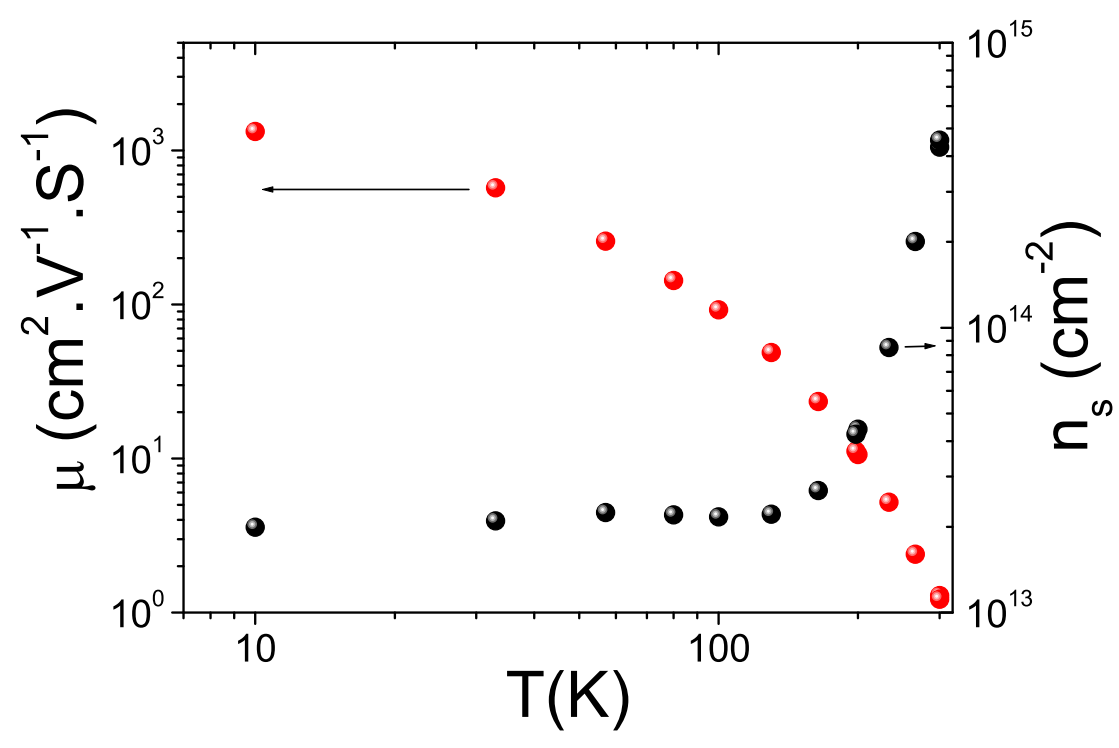

FIG. 5: Mobility and sheet carrier density as a function of temperature, deduced from the Hall measurements using a single carrier model, for the sample grown at $700^{\circ}$. 


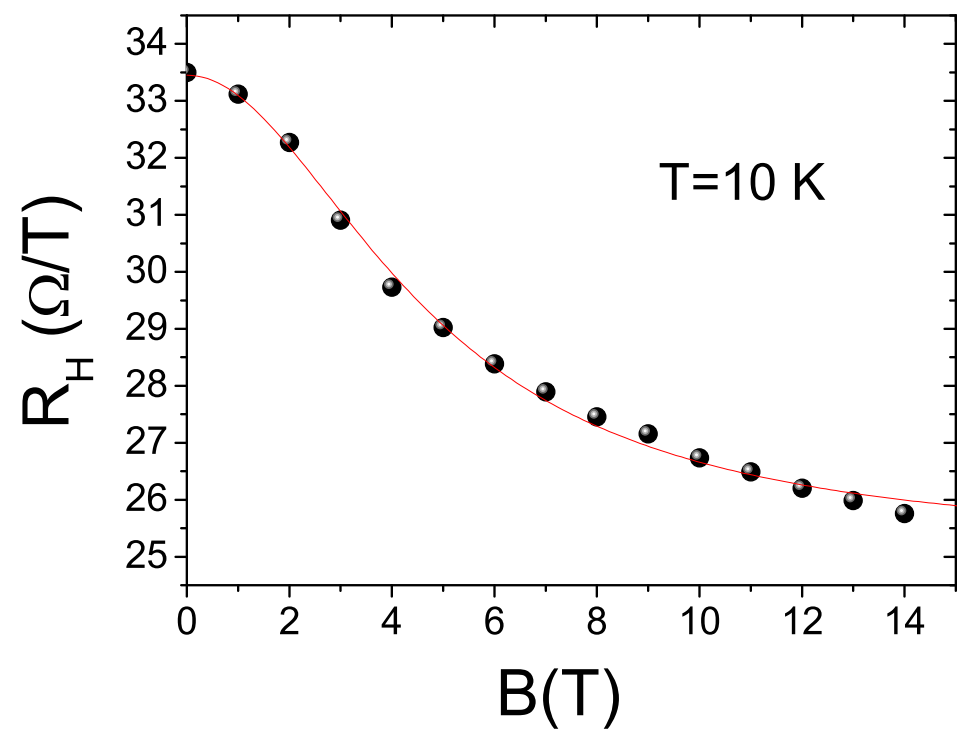

FIG. 6: Hall resistance $\mathrm{R}_{H}=\mathrm{R}_{x y} / \mathrm{B}$ versus the magnetic field at a temperature $\mathrm{T}=10 \mathrm{~K}$ for the sample grown at $700^{\circ}$. The solid line is a fit performed with the two-bands Hall effect (equation (2)).

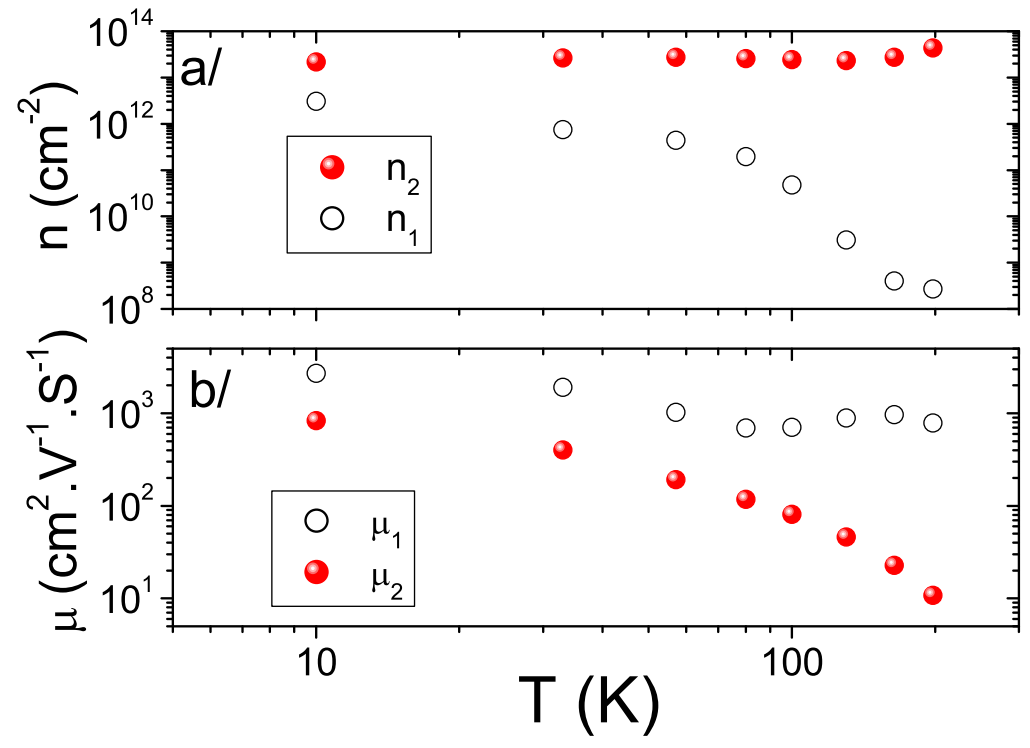

FIG. 7: a/ Sheet carrier densities and b/ mobilities (bottom) of light and heavy carriers as a function of temperature $T$, calculated using a two-bands analysis for the sample grown at $700^{\circ}$. 


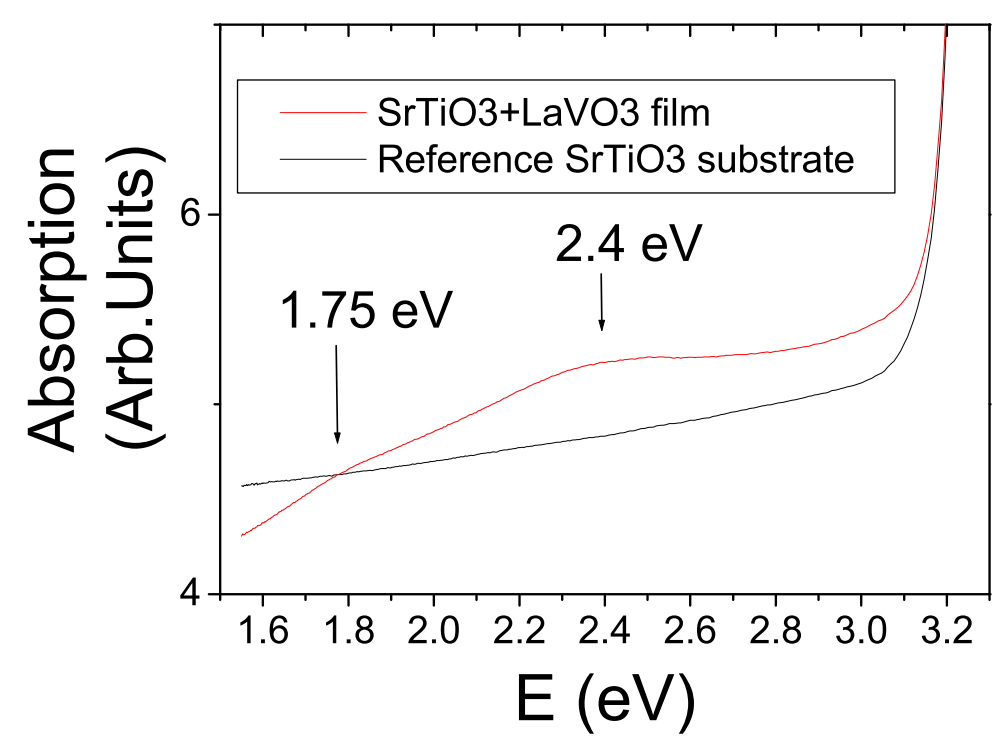

FIG. 8: Optical absorption for the virgin STO substrate and of (LVO/STO) grown at $700^{\circ}$. Note the two broad peaks at 2.4 and $1.75 \mathrm{eV}$ observed only for (LVO/STO), and characteristics of oxygen vacancies in STO.

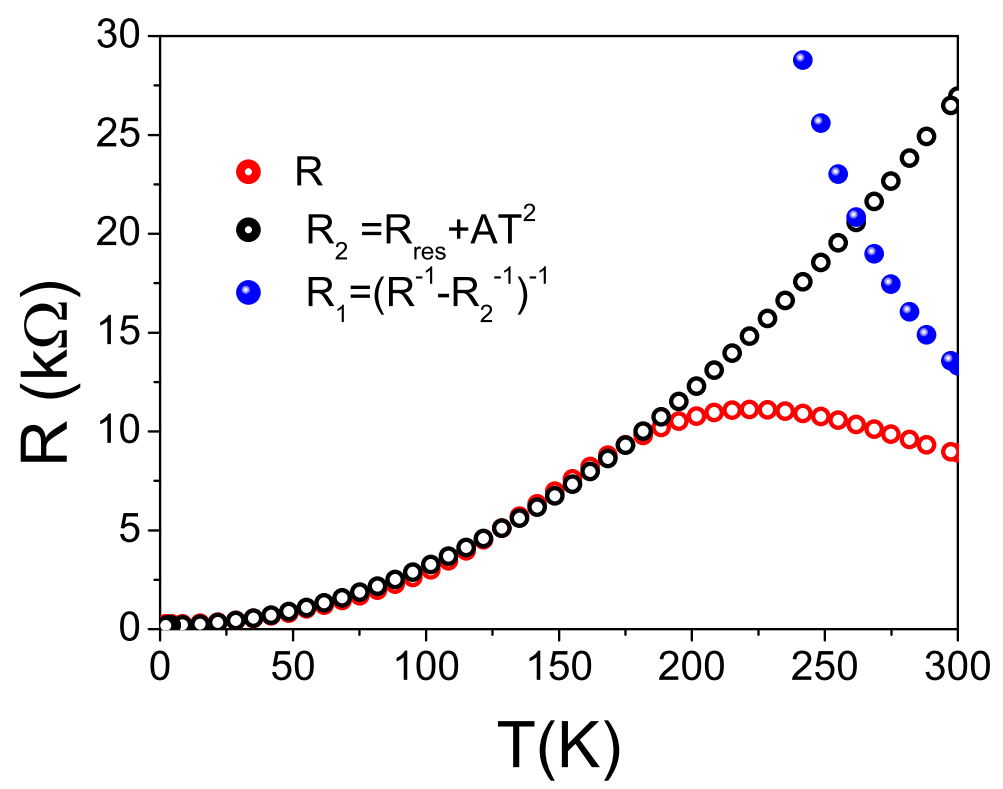

FIG. 9: Variation of the resistance $R$ of the sample grown at $700^{\circ}$ as function of $\mathrm{T}$ temperature. Also shown $\mathrm{R}_{2}=$ $\mathrm{R}_{\text {res }}+\mathrm{AT}^{2}$ corresponding to the STO contribution and the deduced $\mathrm{R}_{1}$ resistance (LVO contribution) using a parallel resistor model. 


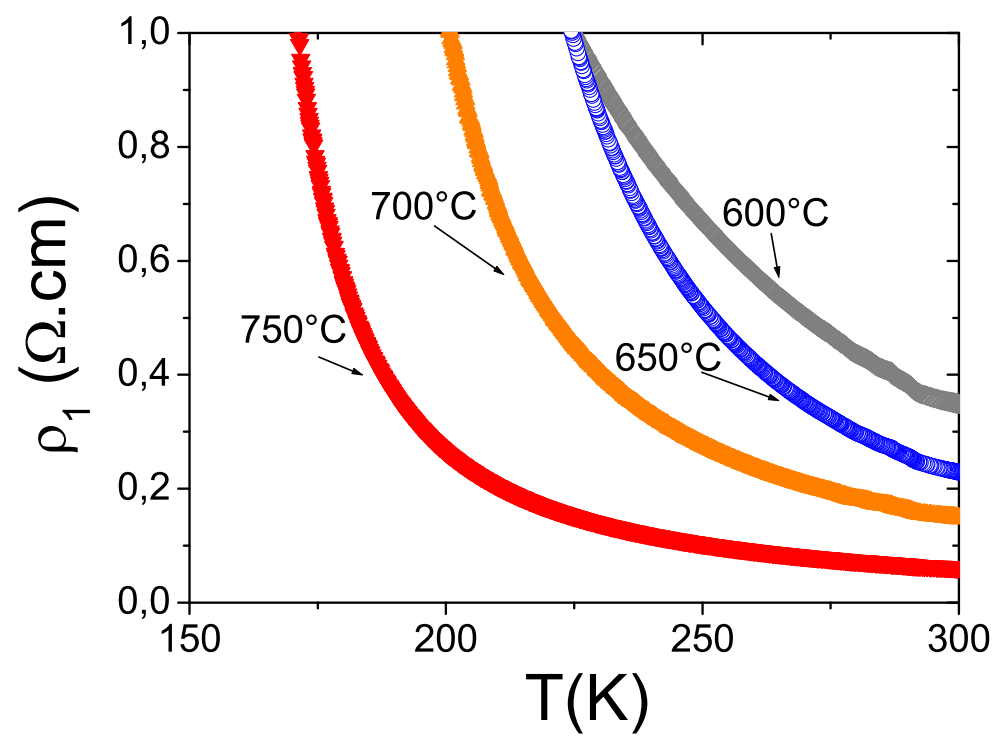

FIG. 10: $\rho_{1}(T)$ deduced from the parallel resistor analysis and corresponding to the LVO component, for the different growth temperatures.

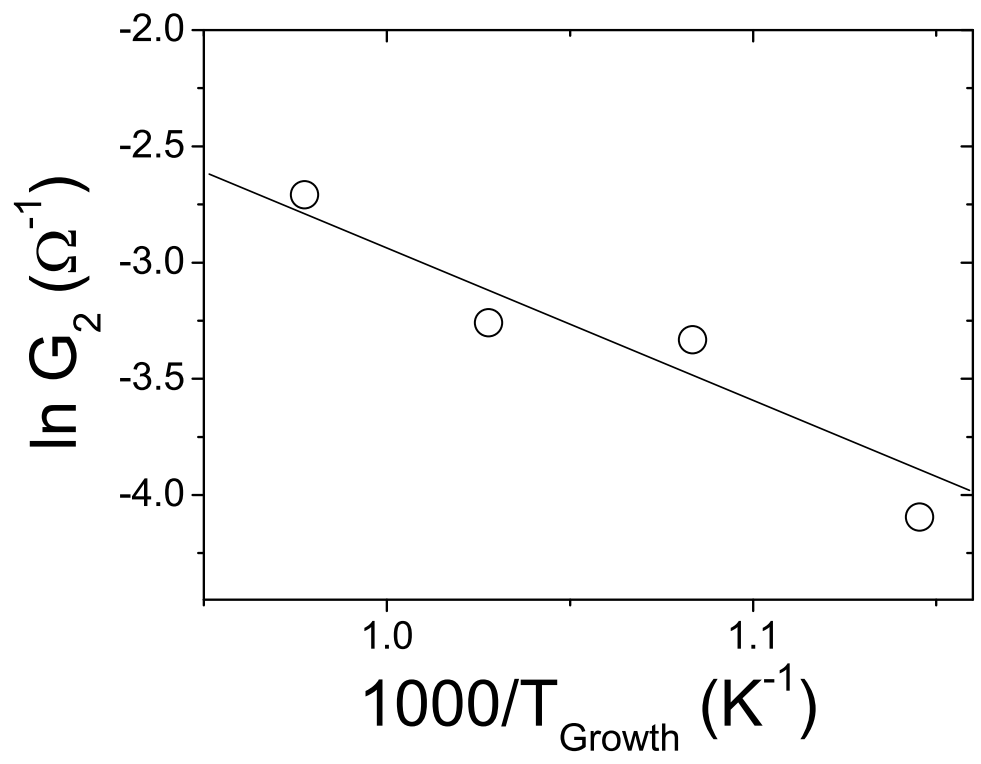

FIG. 11: ln $\mathrm{G}_{2}$ as function of $1000 / \mathrm{T}_{\text {growth }}$. The line corresponds to an activation energy of $\mathrm{U} \sim 0.9 \mathrm{eV}$ (see text for details). 